J. Bourgain, Institute for Advanced Study, Princeton, NJ 08540, USA.

e-mail: bourgain@math.ias.edu

S. Kostyukovsky, School of Mathematical Sciences, Tel Aviv University, Tel Aviv 69978, Israel. e-mail: sergey@post.tau.ac.il

A. Olevskiir, School of Mathematical Sciences, Tel Aviv University, Tel Aviv 69978, Israel. e-mail: olevskii@post.tau.ac.il

\title{
A REMARK ON A MAXIMAL OPERATOR FOR FOURIER MULTIPLIERS
}

\begin{abstract}
For a finite set $\Lambda$ on the circle we consider a family of the multiplier operators $T_{m}$ in $l_{2}(\mathbb{Z})$ generated by the $2^{-m}$-neighborhoods of $\Lambda$. We show that the norm of the corresponding maximal operator $T$ can not be estimated by an absolute constant.
\end{abstract}

Let $T$ be a circle group $\mathbb{R} / Z$ identified in a standard way with the interval $[0,1)$. For $g \in L^{2}(\mathbb{T})$ we denote by $\widehat{g}$ the Fourier transform:

$$
\widehat{g}(k)=\int_{\mathbb{T}} g(t) e^{-2 \pi i k t} d t \quad(k \in \mathbb{Z})
$$

and by $f \mapsto \check{f}$ the inverse operator from $l_{2}(\mathbb{Z})$ onto $L^{2}(\mathbb{T})$.

Given a set $\Lambda \subset \mathbb{T}$ of $N$ distinct points we denote

$$
\Lambda(m)=\bigcup_{\lambda \in \Lambda}\left(\lambda-2^{-m}, \lambda+2^{-m}\right) .
$$

Consider the multiplier operator in $l_{2}(\mathbb{Z})$ :

$$
T_{m}: \quad f \mapsto\left\{\check{f} \chi_{\Lambda(m)}\right\}^{\wedge}
$$

$\left(\chi_{E}\right.$ is an indicator function of the set $\left.\mathrm{E}\right)$

\footnotetext{
Key Words: Fourier multiplier, ergodic theorem, Kolmogorov rearrangement theorem.

Mathematical Reviews subject classification: 42A05, 42A45.

Received by the editors October 13, 2000

* Papers authored or co-authored by a Contributing Editor are managed by a Managing Editor or one of the other Contributing Editors.
} 
and the corresponding maximal operator

$$
M_{\Lambda}: \quad f \mapsto \sup _{m \in Z}\left|\left(T_{m} f\right)(k)\right|
$$

The following inequality was proved in [1] (and used there essentially for the "squares" ergodic theorems) :

$$
\left\|M_{\Lambda} f\right\| \leq C \log ^{2} N\|f\|
$$

(here and below we denote by $C$ positive absolute constants).

It was asked in [1] whether the dependence on $N$ might be removed from the inequality. Here we prove that it can not. Moreover, the log factor in (2) is essentially sharp .

Theorem. For any $N$ there exist a set $\Lambda \subset \mathbb{T}, \quad$ card $\Lambda=N$ and $f \in l_{2}(\mathbb{Z})$, s.t.

$$
\left\|M_{\Lambda} f\right\|>C \log ^{\alpha} N\|f\|
$$

( $\alpha>0$ is an absolute constant; one can take $\alpha=1 / 4)$.

The proof is based on the Kolmogorov "rearrangement" theorem: there exists an $L^{2}$ - Fourier series which diverges (unboundedly) almost everywhere after some permutation of its terms (see [2], ch. 3).

We use the following equivalent form of this theorem: given any $K>0$ one can find a trigonometric polynomial

$$
P(x)=\sum_{j=1}^{N} b_{j} e^{2 \pi i n_{j} x}
$$

such that the conditions below are fulfilled :

$$
\begin{aligned}
& \left\{n_{j}\right\}_{1}^{N} \text { is a rearrangement of }\{1,2, \ldots, N\} ; \\
& \qquad \sum_{j}\left|b_{j}\right|^{2}=1 .
\end{aligned}
$$

If denote

$$
P^{*}(x)=\max _{1 \leq l \leq N}\left|\sum_{j=1}^{l} b_{j} e^{2 \pi i n_{j} x}\right|
$$

then

$$
\operatorname{mes}\left\{x \in \mathbb{T} ; \quad P^{*}(x)>K\right\}>1 / 2
$$


The dependence $K \rightarrow N$ was studied by several authors. The best known (apparently) result [3, Lemma 4] means that one can choose

$$
K=C \log ^{1 / 4} N \quad(N=2,3, \ldots)
$$

Remark. A simple measure theoretic argument shows that there is an appropriate translate of $P$, denoted here again by the same symbol, which satisfies properties (3), (4), (6) and

$$
\operatorname{card}\left\{k \in\{1,2, \ldots, N\}: \quad P^{*}\left(\frac{k}{N}\right)>K\right\}>N / 2 .
$$

Now let $N$ be an integer $>1$, and the corresponding polynomial $P$ is defined.

Set:

$$
\Lambda=\left\{\lambda_{j}\right\}_{1}^{N}, \quad \lambda_{j}=\frac{n_{j}}{N}-\frac{9}{10} 2^{-2 N+j-1} ; \quad \delta=\frac{1}{10} 2^{-2 N} .
$$

For a given $l, 1 \leq l \leq N$ consider the set $\Lambda(2 N-l+1)$ according to (1). Obviously it contains $\delta$-neighborhoods of the points $\left\{\frac{n_{j}}{N}\right\}, 1 \leq j \leq l$ and does not intersects $\delta$-neighborhoods of other points $\left\{\frac{k}{N}\right\}$.

Define for $x \in \mathbb{T}$ :

$$
\begin{gathered}
\psi(x)=\frac{1}{\sqrt{\delta}} \chi_{(-\delta / 2, \delta / 2)}(x) \\
g(x)=\sum_{j=1}^{N} b_{j} \psi\left(x-\frac{n_{j}}{N}\right) .
\end{gathered}
$$

As supports of the summands are disjoint we get from (4):

$$
\|g\|=1 \text {. }
$$

It follows that for $m=2 N-l+1$

$$
g(x) \chi_{\Lambda(m)}(x)=\sum_{j=1}^{l} b_{j} \psi\left(x-\frac{n_{j}}{N}\right),
$$

so for $f=\widehat{g}$ we have:

$$
\left.\left(M_{\Lambda} f\right)(k)=\sup _{m} \mid \widehat{\left(g \chi_{\Lambda(m)}\right.}\right)(k) \mid \geq
$$




$$
\max _{1 \leq l \leq N}\left|\sum_{j=1}^{l} b_{j} \widehat{\psi}(k) e^{2 \pi i \frac{n_{j}}{N} k}\right|=|\widehat{\psi}(k)| P^{*}\left(\frac{k}{N}\right) .
$$

Using the elementary inequality

$$
|\widehat{\psi}(k)|=\frac{1}{\sqrt{\delta}} \frac{\sin \pi \delta k}{\pi k}>\frac{\sqrt{\delta}}{2}, \quad 1 \leq k<\frac{1}{2 \delta}
$$

we get:

$$
\left\|M_{\Lambda} f\right\|^{2} \geq \sum_{1 \leq k<1 /(2 \delta)}|\widehat{\psi}(k)|^{2} P^{*}\left(\frac{k}{N}\right)^{2}>\frac{\delta}{4} \sum_{1 \leq k<1 /(2 \delta)} P^{*}\left(\frac{k}{N}\right)^{2} .
$$

Because of (7) the last sum contains at least $C / \delta$ members exceeding $K^{2}$, so we have:

$$
\left\|M_{\Lambda}\right\|>C K
$$

and the result follows from (6) and (8).

\section{References}

[1] J. Bourgain, Pointwise ergodic theorems on arithmetic sets, Publ. Math. IHES 69.

[2] A. Olevskiř, Fourier Series with Respect to General Orthogonal Systems, Springer-Verlag, 1975.

[3] S. Nakata, On the divergence of rearranged Fourier series of square integrable functions, Acta Sci. Math. 32, 59-70 (1971). 